\title{
Catastrophic Hindfoot Collapse after Tibiotalocalcaneal Nailing: A Case Series
}

\author{
Adam Bernatsky, DPM ${ }^{1}$, Zachery Weyandt, $D P M^{2^{*}}$ and David Seligson, $M^{3}$ \\ ${ }^{1}$ Fellow, Podiatric Medicine and Surgery, Department of Orthopedics, University of Louisville, Louisville, KY, USA \\ ${ }^{2}$ Resident, Podiatric Medicine and Surgery, Department of Orthopedics, University of Louisville, Louisville, KY, USA \\ ${ }^{3}$ Director, Orthopedic Trauma Fellowship, Department of Orthopedics, University of Louisville, Louisville, KY, USA
}

*Corresponding author: Zachery Weyandt, DPM, Resident, Department of Orthopedics, University of Louisville, 4001 Kresge Way Suite 132, Louisville, KY 40207, USA, Tel: 814-515-3440

\begin{abstract}
Ankle and pilon fractures in the elderly population as well as those with multiple co-morbidities are faced with increased surgical complications and higher mortality.

The use of a tibiotalocalcaneal retrograde hindfoot nail without joint preparation has been utilized in this population with promising results. This procedure, however, does not come without complications. It is often difficult to place the nail in the proper position within the calcaneus, talus, and tibia as medial translation is difficult without preparing the joints for fusion. This may contribute to increased nail failure rates and specifically hindfoot fractures. Three cases of hindfoot nail failure are described in patients treated with this technique. The anatomical relationship of the hindfoot without translation under the tibia is not optimal with current nail designs in the authors' opinion. Thus, we believe there are current design flaws associated with the current hindfoot nails and more thought should be given to the spatial relationships of these bones such that a hindfoot nail with a greater valgus bend or new design concept should be entertained.
\end{abstract}

\section{Keywords}

Fragility fracture, Hindfoot nail, Retrograde nail, Tibiotalocalcaneal fusion

\section{Abbreviations}

Tibiotalocalcaneal (TTC)

\section{Introduction}

Ankle and pilon fractures are common injuries of the lower extremity that can have a detrimental impact to geriatric and immunocompromised populations [1,2].
With an increase in elderly and diabetic patients, more patients are presenting with poor bone quality and multiple comorbidities which complicate their injuries [2-4]. Common comorbidities include, but are not limited to; diabetes mellitus, peripheral neuropathy, end stage renal disease, as well as peripheral vascular disease. These patients present a challenge with regard to open reduction and internal fixation due to a high rate of complications [5]. Ankle fractures in these patients have been classified as ankle fragility fractures and there has been podiatric and orthopedic literature describing techniques and outcomes when these types of fractures are treated with retrograde tibiotalocalcaneal (TTC) nails without joint preparation. The TTC arthrodesis to improve walking was described by Professor Eduard Albert as early as 1882 and has been more commonly performed for end stage ankle and subtalar pathology $[6,7]$.

A recent study by Georgiannos, et al. found that elderly patients treated with tibiotalocalcaneal nails had a decreased mortality rate, higher rate of return to activity, decreased complications, and shorter hospital stays [8]. It has been discussed that these patients are also able to weight-bear immediately following surgery. Elderly patients are often forced to remain non weight-bearing after conventional open reduction internal fixation, which then increases the risk for perioperative and post-operative complications such as pneumonia, deep vein thrombosis, and pressure sores [9].

Ebaugh, et al. recently introduced the extended in- 
clusion criteria for TTC nailing without joint preparation in unstable ankle and pilon fractures for patients with complicated diabetes. They defined complicated diabetics as those that have confirmed diabetes along with one or more of the following: Neuropathy, nephropathy, and peripheral vascular disease. Similar to fragility fractures addressed with this surgical technique, treatment goals are comparable to those stated above with emphasis on limb salvage. Serious complications have been reported as high as $43 \%$ in this population of patients with traditional surgical intervention of ankle fractures [2]. A minimally invasive approach with a stable construct for early ambulation is ideal for complicated diabetics.

Hindfoot fractures after retrograde intramedullary nailing of the ankle are a complication of this procedure. Three cases highlighting these complications are reported (Table 1). These fractures are caused by insertion of nails that do not conform to the anatomy of the hindfoot. As the use of hindfoot nailing increases, this complication will doubtless continue until better implants are devised.

\section{Surgical Technique}

The entrance point which is 1.5 to $2 \mathrm{~cm}$ proximal to the calcaneo cuboid joint is localized fluoroscopically. This is in line with the axis of the tibial shaft. An awl is inserted through the calcaneus and into the talus. Placement of the awl is verified by the image intensifier. The awl is introduced retrograde through the scar of the tibial physis. A ball tip wire is exchanged with the awl and sequential reaming by $0.5 \mathrm{~mm}$ is commenced for preparation of a $200 \mathrm{~mm}$ supracondylar femur nail. The intramedullary canal is reamed to a diameter $2 \mathrm{~mm}$ greater than the diameter of the nail. The nail is pushed into place, never hammered. Two distal screws are placed utilizing the external guide from lateral to medial, one in the talus and one in the calcaneus. Two tibial shaft screws are then inserted using the external guide as well. Patients are allowed to bear weight immediately.

\section{Case Series}

\section{Case 1}

A 56-year-old male who sustained a right bimalleolar ankle fracture in a trip and fall outside. He is an in-

Table 1: Summary of Patients with Hindfoot Nail Failure.

\begin{tabular}{|l|l|l|l|l|}
\hline Age & Indication & Comorbidities & Time to Failure & Outcome \\
\hline $56 \mathrm{M}$ & Bimalleolar Ankle Fracture & IDDM, peripheral neuropathy, PVD & 18 days & $\begin{array}{l}\text { Removal of nail with } \\
\text { pinning of ankle joint }\end{array}$ \\
\hline $64 \mathrm{~F}$ & Trimalleolar Ankle Fracture & $\begin{array}{l}\text { IDDM, ESRD, peripheral neuropathy, } \\
\text { PVD, CVA }\end{array}$ & 4 months & Splinted NWB \\
\hline $66 \mathrm{M}$ & Pilon Fracture & IDDM, peripheral neuropathy, CKD, PAD & 4 months & Revision of nail \\
\hline
\end{tabular}

*IDDM: Insulin Dependent Diabetes Mellitus; PAD: Peripheral Vascular Disease; ESRD: End Stage Renal Disease; CKD: Chronic Kidney Disease; CVA: Cerebral Vascular Accident; DVT: Deep Vein Thrombosis, CABG: Coronary Artery Bypass Graft.

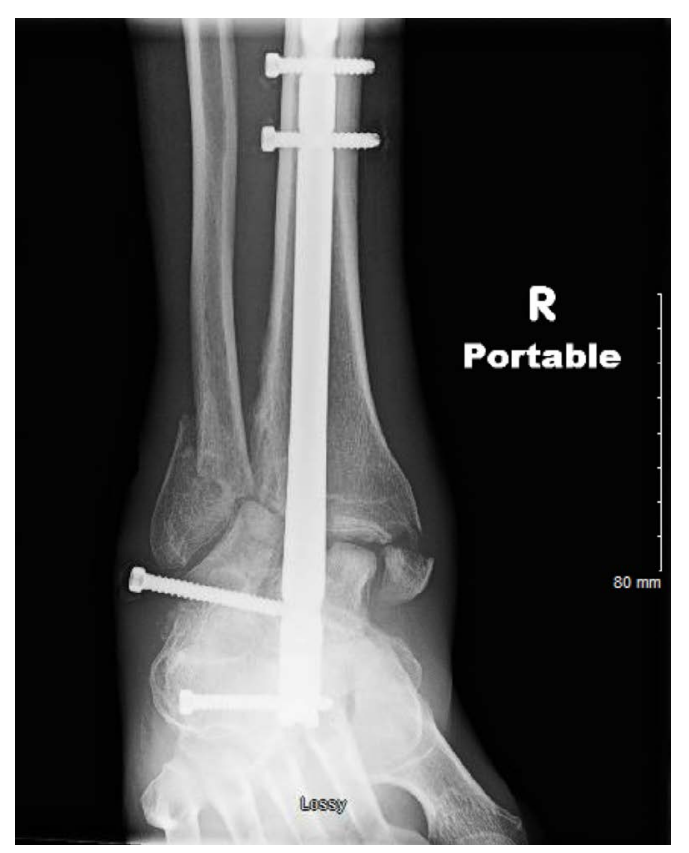

(A)

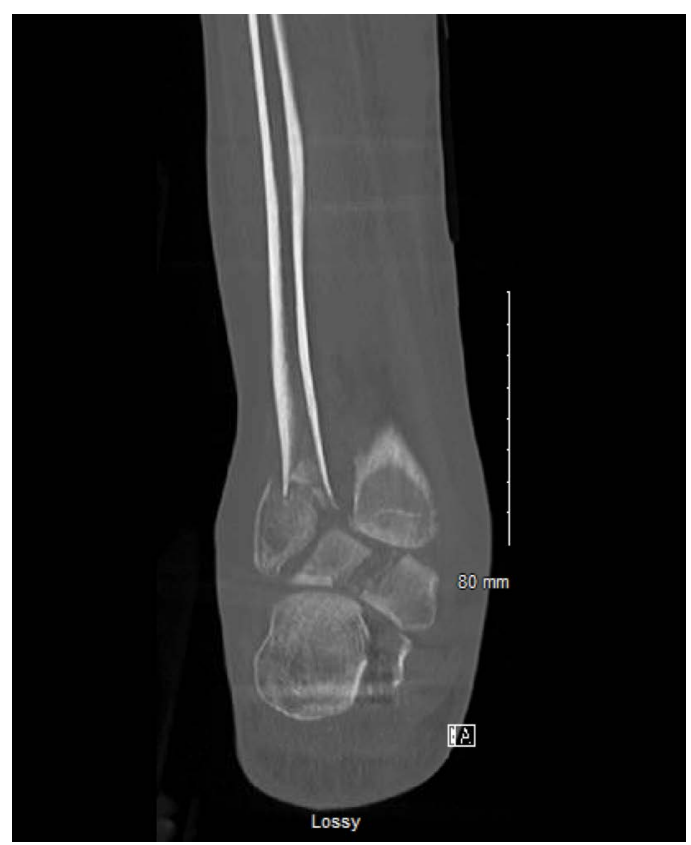

(B)

Figure 1: (A) AP radiograph of hindfoot fractures after retrograde hindfoot nailing and; (B) Post-operative coronal CT views depicting non-union of the fibula in addition to fracture of the talus and calcaneus after the nail is explanted. 


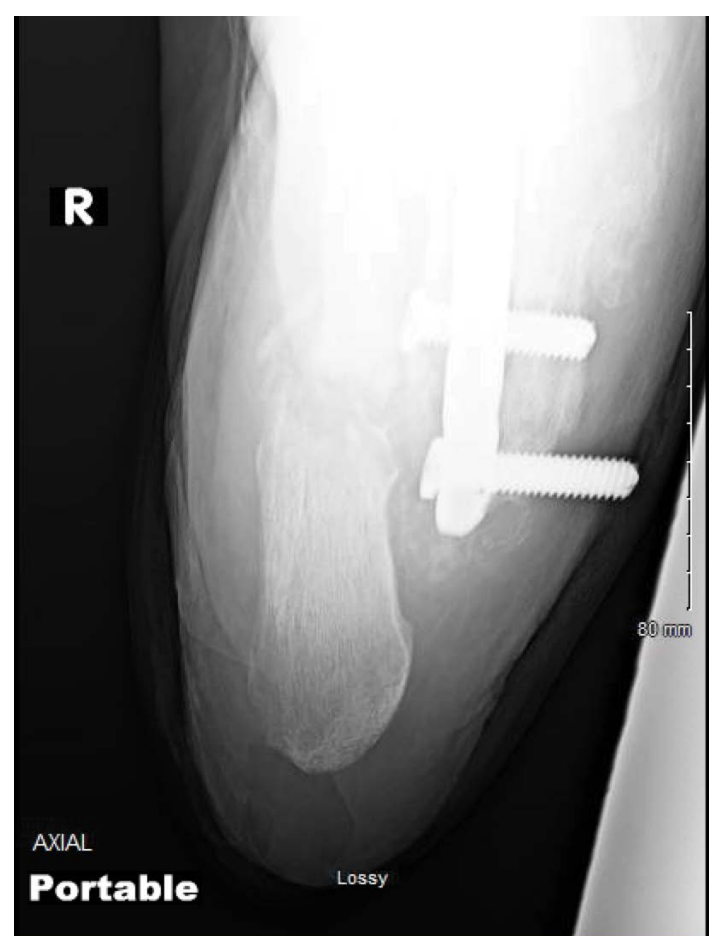

(A)

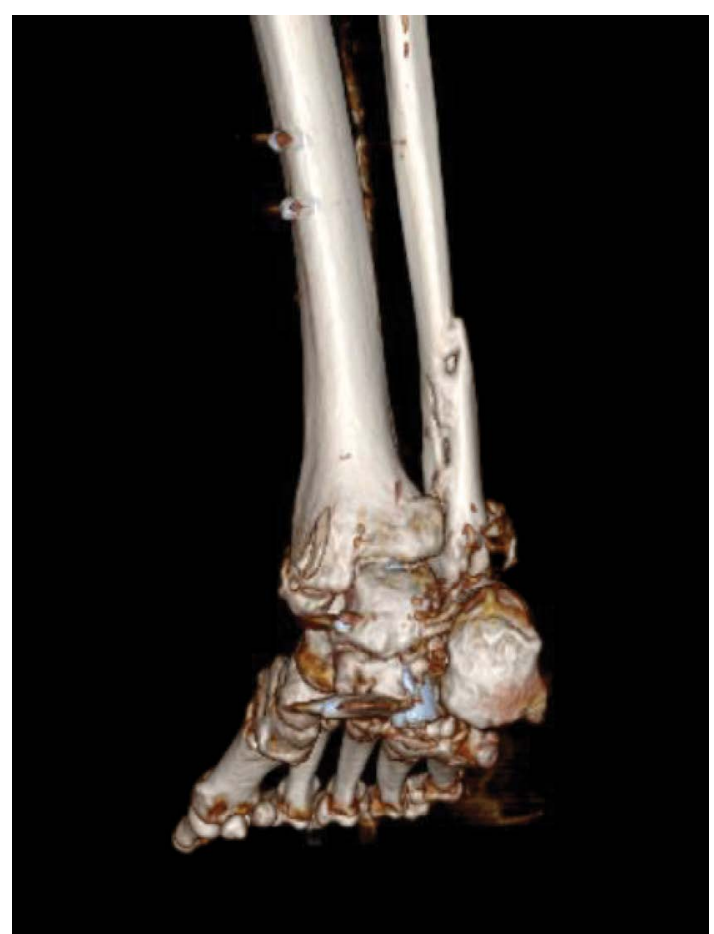

(B)

Figure 2: (A) Post-operative failure of hindfoot nail (Calcaneal axial radiograph); (B) 3D reconstruction image of fractured calcaneus.

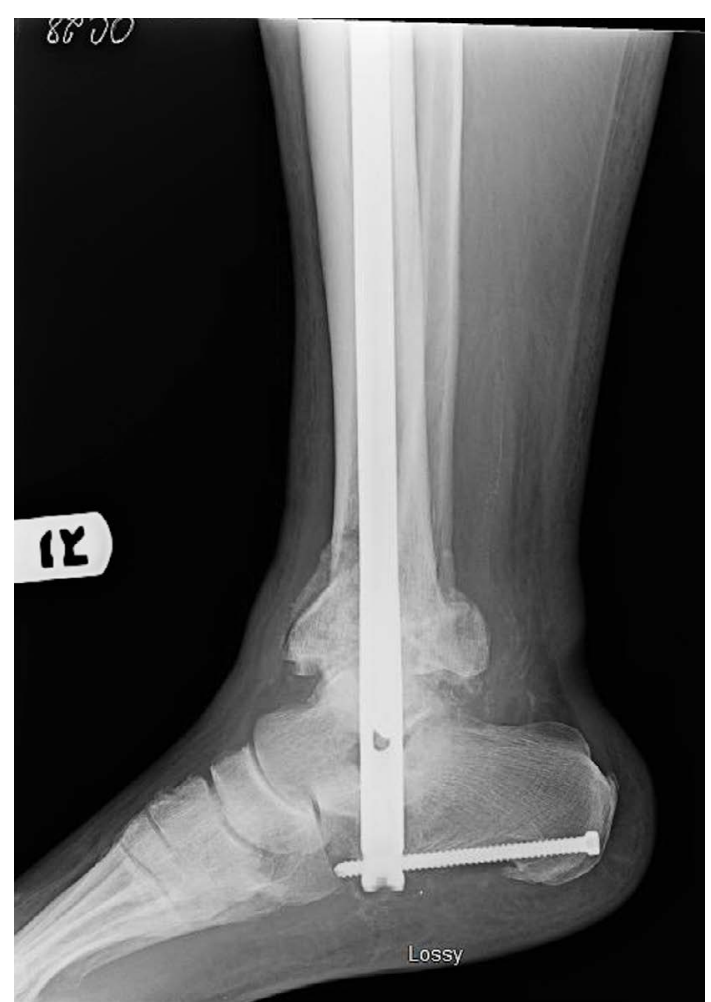

Figure 3: Post-operative failure of hindfoot nail. Lateral radiograph before revision surgery showing hardware failure.

sulin dependent diabetic with an elevated $\mathrm{HbA1c}$ and peripheral neuropathy. He has chronic lower extremity wounds due to vascular disease. His past medical history includes hypertension, depression and acute kidney injury. He was treated with a hindfoot nail and the postoperative course was uneventful until approximately 18 days later the patient presented with loosening of the implant and fractures of both the talus and calcaneus (Figure 1).

\section{Case 2}

A 64-year-old female who sustained a trimalleolar fracture after falling at home. Patient has a past medical history consisting of end stage renal disease (undergoing dialysis), insulin dependent diabetes, peripheral neuropathy, hypertension, peripheral vascular disease, and history of previous cerebrovascular accident. She was initially treated with a hindfoot nail and was able to ambulate post operatively. Patient returned 4 months later with an ulceration and prominent screw from the medial foot. Upon viewing the radiographs, it was noted that her hindfoot nail had fractured through her calcaneus (Figure 2).

\section{Case 3}

A 64-year-old male who sustained a right closed piIon fracture secondary to a ladder fall. $\mathrm{He}$ is an insulin dependent diabetic with peripheral neuropathy, chronic kidney disease, and peripheral arterial disease. He presented to the clinic in a malreduced position with a medial malleolus fracture blister after first being assessed by an outside facility. He initially was treated with closed reduction and splinting. The patient underwent closed reduction and placement of a retrograde hindfoot nail three weeks later. He developed a painful non-union and experienced hardware failure (Figure 3 ), which then required hindfoot nail removal and revision with a larger diameter nail at 4 months. 


\section{Discussion}

The placement of a retrograde hindfoot nail is complicated by the anatomical relationship of the hindfoot and tibia. The typical insertion of retrograde hindfoot nails in the scenario of joint preparation of the ankle and subtalar joint rely on the ability to shift the hindfoot (talus/calcaneus complex) beneath the tibia. However, when the joints are not prepared as in cases of traumatic ankle and pilon fractures, this medialization is not possible. Anatomical studies have shown the calcaneal midsection lies between 5-10 mm lateral the tibial anatomical axis [10]. The accepted practice of TCC fusion with a retrograde nail includes medial translation of the hindfoot to properly align the calcaneus to the tibia [11]. McGarvey showed in addition to proper alignment, shifting the calcaneus a mean of $9.3 \mathrm{~mm}$ medially reduces injury to the lateral plantar neurovascular bundle [12].

The structural anatomy of the talus in relation to the calcaneus is not a true linear relationship. The body of the talus lies slightly medial to that of the body of the calcaneus which causes additional hardship for placement of an intramedullary nail. The calcaneus is a pretzel shaped bone with a twist and lies slightly lateral to the body of the talus. One way to overcome this alignment is to bring the nail posterior from the tuberosity of the calcaneus as shown by Klaue, et al. [13]. Fractures in the hindfoot occur as there is not a nail which specifically matches the anatomic relationship between the talus, calcaneus, and tibia without translating the hindfoot. Further, the typical insertion site of the calcaneus in order to place the nail centrally in the tibial canal is at the junction of the sustentaculum and medial wall of the calcaneus in cases of nail insertion without joint preparation. This location results in less bone substance around the nail and is a less stable construct. There are several different nails utilized for TTC intramedullary nailing. Surgeon preference dictates nail selection. The use of a femoral supracondylar nail with a distal four degree bend to accommodate for the spatial relationship between the calcaneus and talus has been utilized in our reported cases.

Several studies have reported successful outcomes for primary TCC nailing for fragility ankle fractures and only one for ankle fractures of the complicated diabetic. Ebaugh, et al. performed a retrospective study of $27 \mathrm{pa}-$ tients that underwent primary TTC nailing for unstable ankle fractures without joint preparation. Their series included patients with complicated diabetes. Complicated diabetes was defined as those with diabetes in addition to one or more of the following: Neuropathy, nephropathy, and/or peripheral vascular disease. The study group has a mean age of 66 years, a body mass index of 38 , and a hemoglobin A1c of $7.4 \%$. The limb salvage rate was reported to be $96 \%$ and the mean time to weight bearing was 6.7 weeks. They reported a surgical complication rate of $18 \%$. There were three hardware failures with only one requiring revision surgery consisting of formal joint preparation and TCC nailing for arthrodesis. Out of the 27 patients in the study group, three underwent implant removal. The ability to ambulate was maintained in $81 \%$ of patients [2]. The cases reported include TTC nailing for unstable ankle and pilon fractures in similar patients. Mean time to weight bearing, however, is sooner than 6.7 weeks post-operatively.

Jonas, et al. reported a retrospective study of 31 geriatric patients with unstable acute ankle fractures treated with a hindfoot nail without joint preparation. A $94 \%$ rate of return to pre-injury ambulation was reported. Complications included three periprosthetic fractures that occurred adjacent to the proximal tip of the nail as well as two instances of nail breakage. The nail length was not reported [9]. Al-Nammari, et al. reported a retrospective study of 48 geriatric patients treated with a long TCC nail without joint preparation for unstable ankle fractures. Each hindfoot nail was long enough to pass the tibial isthmus. The pre-injury functionality was achieved in $90 \%$ of patients and there were no cases of nail breakage or periprosthetic fractures. Three patients experienced at least one loosened or broken screw and one case of a below knee amputation was reported [14]. Baker, et al. also described the use of a long TCC nail in 16 patients reporting no implant failure or periprosthetic fractures [15].

\section{Conclusion}

Splinting unstable ankle and pilon fractures with TTC nails reduce morbidity not only for geriatric patients with fragility fractures but also for those with diabetes and additional comorbidities [1,2]. It is the authors' opinion that there is an increased risk of hindfoot fractures and nail failure due to the inability to medialize the hindfoot under the tibia when there is no joint preparation. The inability to center the hindfoot under the tibia increases the difficulty of placing the nail in adequate bone of the calcaneus which risks fracture and failure. This paper is a report of three such cases of hindfoot failure. It is our opinion that a redesigned retrograde hindfoot nail with more valgus bend and tunnels for screws directed towards the calcaneocuboid joint and constant fragment would improve this procedure. A straight or minimal valgus bent nail is more likely to cause a fracture than a nail with more valgus bend or other improved design for these fractures.

\section{Acknowledgements}

None.

\section{Financial Disclosure}

None reported.

\section{Conflict of Interest}

David Seligson, MD is a consultant for Stryker Trauma. 


\section{Authors Contribution}

All authors were fully involved in the study and equally participated in the manuscript.

\section{References}

1. Taylor BC, Hansen DC, Harrison R, Lucas DE, Degenova D (2016) Primary Retrograde Tibiotalocalcaneal Nailing For Fragility Ankle Fractures. lowa Orthop J 36: 75-78.

2. Ebaugh MP, Umbel B, Goss D, Taylor BC (2019) Outcomes of Primary Tibiotalocalcaneal Nailing for Complicated Diabetic Ankle Fractures. Foot Ankle Int 40: 1382-1387.

3. Nilsson G, Jonsson K, Ekdahl C, Eneroth M (2007) Outcome and quality of life after surgically treated ankle fractures in patients 65 years or older. BMC Musculoskelet Disord 8: 127.

4. Boyle JP, Honeycutt AA, Narayan KM, Hoerger TJ, Geiss LS, et al. (2001) Projection of diabetes burden through 2050: Impact of changing demography and disease prevalence in the U.S. Diabetes Care 24: 1936-1940.

5. Litchfield JC (1987) The treatment of unstable fractures of the ankle in the elderly. Injury 18: 128-132.

6. Albert Eduard (1882) Einige Fälle von künstlicher Ankzlosen bildung an paralytischen Gleidmassen. Weiner Medizinische Press.

7. Dujela M, Hyer CF, Berlet GC (2018) Rate of subtalar joint arthrodesis after retrograde tibiotalocalcaneal arthrodesis with intramedullary nail fixation: Evaluation of the rain database. Foot Ankle Spec 11: 410-415.
8. Georgiannos D, Lampridis V, Bisbinas I (2017) Fragility fractures of the ankle in the elderly: Open reduction and internal fixation versus tibio-talo-calcaneal nailing: Short-term results of a prospective randomized-controlled study. Injury 48: 519-524.

9. Jonas SC, Young AF, Curwen CH, McCann PA (2013) Functional outcome following tibio-talar-calcaneal nailing for unstable osteoporotic ankle fractures. Injury 44: 994997.

10. Roukis TS (2006) Determining the insertion site for retrograde intramedullary nail fixation of tibiotalocalcaneal arthrodesis: A radiographic and intraoperative anatomical landmark analysis. J Foot Ankle Surg 45: 227-234.

11. Belczyk R, Sung W, Wukich D (2008) Technical tip: A simple method for proper placement of an intramedullary nail entry point for tibiotalocalcaneal or tibiocalcaneal arthrodesis. The Foot \& Ankle Journal.

12. McGarvey WC, Trevino SG, Baxter DE, Noble PC, Schon LC (1998) Tibiotalocalcaneal arthrodesis: Anatomic and technical considerations. Foot Ankle Int 19: 363-369.

13. Klaue K, Wichelhaus A, Maik P, Mittlmeier T (2019) The circular arc shaped nail for fixing the tibiotalocalcaneal arthrodesis. After clinical results. Injury 50: 23-31.

14. Al-Nammari SS, Dawson-Bowling S, Amin A, Nielsen D (2014) Fragility fractures of the ankle in the frail elderly patient: Treatment with a long calcaneotalotibial nail. Bone Joint J 96-B: 817-822.

15. Baker G, Mayne AIW, Andrews C (2018) Fixation of unstable ankle fractures using a long hindfoot nail. Injury 49: 2083-2086. 